\title{
Análise da certificação FSC em manejo e cadeia de custódia florestal entre o período de 2012 a 2018
}

\author{
Pompeu Paes Guimarães ${ }^{1 *}$, João Lucas Rebouças de Oliveira ${ }^{1}$, Narjara Walessa Nogueira de Freitas ${ }^{1}$, Daniel \\ Pena Pereira ${ }^{2}$, Luciélia Lacerda da Silva ${ }^{1}$
}

\begin{abstract}
RESUMO: O objetivo deste artigo foi analisar o quantitativo de empreendimentos certificados pelo Forest Stewardship Council (FSC) quanto ao manejo florestal e cadeia de custódia florestal (CoC), no período de 2012 a 2018. Com base em dados secundários aplicou-se estatísticas descritas e as médias das operações de manejo florestal certificado e dos certificados de cadeia de custódia florestal anual foram comparadas, quando apresentaram diferenças, pelo teste de Tukey a 5\% de significância. Foram ajustadas modelos de tendência para analisar a taxa anual do número e área de certificados em manejo florestal e do número de certificados para cadeia de custódia. Para os meses avaliados não houve diferença significativas para as variáveis área e número dos empreendimentos certificados em manejo florestal e para o número dos certificados em cadeia de custódia. Quando se comparou as mesmas variáveis agora anualmente, houve diferença significativa. Para a área florestal manejada as maiores extensões, em média, foram relacionais aos anos de 2013, 2015, 2017 e 2018. Para o número de certificados em manejo florestal, em média, se concentrou em 2018. E para o número de certificados em cadeia de custódia, no período de 2015 a 2016. Houve crescimento do quantitativo de empreendimentos certificados FSC em manejo florestal $(5,50)$ e cadeia de custódia $(2,37)$. No entanto, mesmo com tendência de aumento no número de certificados quanto ao manejo florestal houve redução no tamanho das áreas manejadas $(-0,26)$, resultando em áreas menores, em cerca de $21.888,5$ ha.
\end{abstract}

Palavras-chave: área, número, produção florestal

\section{Analysis of FSC certification in forest management and chain of custody from 2012 to 2018}

\begin{abstract}
The main idea of this article was to analyze the quantity of enterprises certified by the Forest Stewardship Council (FSC) regarding forest management and forestry chain of custody (CoC), in the period from 2012 to 2018. Based on secondary data, statistics described and the means of certified forest management operations and annual forest chain of custody certificates were compared, when they differed, by the Tukey test at 5\% significance. Trend models were adjusted to analyze the annual rate of the number and area of certificates in forest management and the number of certificates for the chain of custody. For the months evaluated, there was no significant difference for the variables area and number of enterprises certified in forest management and for the number of certificates in the chain of custody. When comparing the same variables annually now, there was a significant difference. For the managed forest area, the largest extensions, on average, were related to the years 2013, 2015, 2017 and 2018. For the number of certificates in forest management, on average, it was concentrated in 2018. And for the number of chain certificates from 2015 to 2016. There was an increase in the number of FSC certified enterprises in forest management $(5,50)$ and chain of custody $(2,37)$. However, even with an increasing trend in the number of certificates for forest management, there was a reduction in the size of managed areas $(-0,26)$, resulting in smaller areas, around $21.888,5$ ha.
\end{abstract}

Keywords: area, number, forest production

\section{INTRODUÇÃO}

$\mathrm{O}$ processo pelo qual uma organização busca reconhecimento, da sociedade e de seus clientes, de que seus produtos são provenientes de um povoamento manejado segundo padrões e critérios ambientalmente corretos, socialmente justos e economicamente viáveis é conhecido como certificação florestal (PORTAL DA EDUCAÇÃO, 2012).

\footnotetext{
Recebido em 14/04/2020; Aceito para publicação em 23/06/2020

${ }^{1}$ Universidade Federal Rural do Semi-Árido

${ }^{2}$ Instituto Federal do Triângulo Mineiro

*E-mail: pompeu.guimaraes@ufersa.edu.br
}

O processo de certificação florestal pode ocorrer nas modalidades de manejo florestal - afirmação de um sistema de gestão sustentável da floresta - e da cadeia de custódia florestal - que tem por objetivo a identidade e rastreabilidade do produto da floresta em toda a etapa do processo (SPATHELF, MATTOS e BOTOSSO, 2004).

Por avaliações nos relatórios de unidade de manejo, em 2007, Basso et al. (2011a), Basso et al. 
(2011b) em relação ao princípio 1 "Obediência às leis e princípios do FSC", elencaram as principais não conformidades elencadas como problemas com trabalhadores terceirizados e a com a legislação ambiental referentes às áreas de preservação permanente e falta de autorizações de órgãos ambientais.

A obtenção da certificação de cadeia de custódia de produtos de base florestal é condicionada ao atendimento de uma série de requisitos normativos. Cada não conformidade apresentada é documentada para que a organização tome providências quanto à sua correção (TECPAR, 2017).

Como Pereira et al. (2017) demonstraram as mudanças para adequar uma serraria para que pudesse ser certificada em cadeia de custódia FSC pontuando a necessidade de readequação de atividades, organização de notas, fatores de conversão, leis trabalhistas e normas regulamentadores.

Para grandes empresas como a Klabim, em uma avaliação do processo de certificação e resertificação da empresa, Paiva et al. (2015) concluíram que a certificação agregou valor para a gestão operacional da empresa, com vantagens socioeconômicas - mãode-obra, serviços e novos projetos; sociais - canais de comunicação; e ambiental - gerenciamento de recursos hídricos.

$\mathrm{O}$ pequeno produtor na tentativa de certificar seu manejo florestal, além da possibilidade de certificação individual, pode participar da certificação em grupo. Com isso, Basso et al. (2011c), verificaram que apenas oito processos de certificação em grupo ocorreram entre o período de 2002 a 2010, sendo sete em floresta nativa e um em plantada, com principais dificuldades ligadas as áreas ambientais e trabalhistas.

Partindo desse pressuposto objetivou-se analisar o quantitativo de empreendimentos certificados pelo FSC quanto ao manejo florestal e cadeia de custódia florestal entre os anos de 2012 a 2018.

\section{MATERIAL E MÉTODOS}

A certificação florestal brasileira pode ocorrer na modalidade de manejo florestal ou de cadeia de custódia florestal. Com base em dados secundários, disponíveis nos relatórios do FSC, entre o período de
2012 a 2018 (FSC, 2021), filtrou-se os certificados quanto ao número e áreas em manejo florestal e quanto ao número de certificados em cadeia de custódia mensal e anual.

Além da aplicação de estatísticas descritas como média, desvio padrão e coeficiente de variação, o número e a área de operações de manejo florestal certificados e o número de certificados de cadeia de custódia foram submetidos a uma análise de variância no delineamento inteiramente casualizado. As médias das operações de manejo florestal certificado e dos certificados de cadeia de custódia florestal anual foram comparadas pelo teste de Tukey a 5\% de probabilidade, quando estes apresentaram diferenças estatísticas significativas.

Foram ajustados modelos de tendência para analisar a taxa anual do número e área de certificados em manejo florestal e número de certificados de cadeia de custódia, baseando-se em Guimarães et al. (2018); Aguiar et al. (2014); Almeida et al. (2009); Gujarati (2006), conforme Equação 1, 2 e 3.

$$
\begin{aligned}
\operatorname{Ln} Q & =\beta_{0}+\beta_{1} T+\varepsilon \\
\operatorname{Ln} A & =\beta_{0}+\beta_{1} T+\varepsilon \\
r & =e^{\left(\beta_{1}-1\right) 100}
\end{aligned}
$$

em que: $\mathrm{Q}=$ número no ano $\mathrm{t} ; \mathrm{A}=$ área (ha) no ano $\mathrm{t}$; $\mathrm{T}=$ variável tendência, em anos; $\varepsilon=$ termo de perturbação; $r$ = taxa composta.

\section{RESULTADOS E DISCUSSÃO}

As estatísticas descritivas e taxa composta para os empreendimentos certificados FSC em manejo florestal (número e área) e cadeia de custódia (número), são apresentados na Tabela 1.

As taxas compostas podem ser analisadas na Tabela 1. Os empreendimentos certificados em número, tanto para manejo florestal quanto para cadeia de custódia mostraram taxas positivas. Já a certificação em manejo florestal por área foi decrescente no período de 2012 a 2018. Dessa forma é possível perceber que o número de empreendimentos certificados em manejo florestal aumentou, no entanto, as áreas individuais reduziram. 
Tabela 1. Estatísticas descritivas dos empreendimentos certificados FSC em manejo florestal e cadeia de custódia florestal.

\begin{tabular}{|c|c|c|c|c|c|c|c|c|c|}
\hline \multirow{2}{*}{$\begin{array}{l}\text { Méd } \\
\left(\mathrm{CV}_{\%}\right)\end{array}$} & \multicolumn{3}{|c|}{ Manejo Florestal } & \multirow{2}{*}{$\begin{array}{c}\mathrm{CoC} \\
\mathrm{N}\end{array}$} & \multirow{2}{*}{$\begin{array}{l}\text { Méd } \\
\left(\mathrm{CV}_{\%}\right)\end{array}$} & \multicolumn{3}{|c|}{ Manejo Florestal } & \multirow{2}{*}{$\begin{array}{c}\text { Coc } \\
\mathrm{N}\end{array}$} \\
\hline & Área & $\mathrm{N}$ & Área/N & & & Área & $\mathrm{N}$ & Área/N & \\
\hline Jan & $\begin{array}{c}6.303 .503,9 \\
(11,6)\end{array}$ & $\begin{array}{l}102,0 \\
(12,4)\end{array}$ & $61.799,1$ & $\begin{array}{c}1.002,1 \\
(9,4)\end{array}$ & 2012 & $\begin{array}{c}6.114 .086,9 \mathrm{~b} \\
(14,5)\end{array}$ & $\begin{array}{c}84,8 \mathrm{f} \\
(4,8)\end{array}$ & $72.100,1$ & $\begin{array}{c}880,9 \mathrm{~d} \\
(3,4)\end{array}$ \\
\hline Fev & $\begin{array}{c}6.462 .289,7 \\
(14,1)\end{array}$ & $\begin{array}{l}102,4 \\
(13,0)\end{array}$ & $63.108,3$ & $\begin{array}{c}997,9 \\
(9,2)\end{array}$ & 2013 & $\begin{array}{c}7.316 .191,2 \mathrm{a} \\
(0,9)\end{array}$ & $\begin{array}{c}95,6 \mathrm{e} \\
(2,8)\end{array}$ & $76.529,2$ & $\begin{array}{c}965,3 \mathrm{c} \\
(1,2)\end{array}$ \\
\hline Mar & $\begin{array}{c}6.448 .013,6 \\
(14,3)\end{array}$ & $\begin{array}{l}101,3 \\
(12,6)\end{array}$ & $63.652,7$ & $\begin{array}{c}1.001,6 \\
(8,7)\end{array}$ & 2014 & $\begin{array}{c}6.699 .247,2 \mathrm{~b} \\
(8,1)\end{array}$ & $\begin{array}{c}103,3 \mathrm{~d} \\
(1,7)\end{array}$ & $64.852,3$ & $\begin{array}{c}1.027,0 \mathrm{~b} \\
(1,4)\end{array}$ \\
\hline Abr & $\begin{array}{c}6.258 .738,3 \\
(11,3)\end{array}$ & $\begin{array}{l}102,9 \\
(12,3)\end{array}$ & $60.823,5$ & $\begin{array}{c}1.005,3 \\
(8,5)\end{array}$ & 2015 & $\begin{array}{c}6.307 .569,9 a b \\
(3,1)\end{array}$ & $\begin{array}{c}102,4 \mathrm{~d} \\
(2,9)\end{array}$ & $61.597,4$ & $\begin{array}{c}1.070,8 \mathrm{a} \\
(0,6)\end{array}$ \\
\hline Mai & $\begin{array}{c}6.484 .041,0 \\
(5,8)\end{array}$ & $\begin{array}{l}103,7 \\
(12,1)\end{array}$ & $62.526,9$ & $\begin{array}{c}1.010,9 \\
(7,4)\end{array}$ & 2016 & $\begin{array}{c}6.161 .373,6 \mathrm{~b} \\
(1,3)\end{array}$ & $\begin{array}{c}110,1 \mathrm{c} \\
(2,0)\end{array}$ & $55.961,6$ & $\begin{array}{c}1.086,6 \mathrm{a} \\
(1,9)\end{array}$ \\
\hline Jun & $\begin{array}{c}6.598 .581,0 \\
(5,9)\end{array}$ & $\begin{array}{l}104,3 \\
(11,8)\end{array}$ & $63.265,4$ & $\begin{array}{c}1.012,3 \\
(6,8)\end{array}$ & 2017 & $\begin{array}{c}6.525 .880,9 \mathrm{ab} \\
(6,1)\end{array}$ & $\begin{array}{c}114,8 \mathrm{~b} \\
(1,7)\end{array}$ & $56.845,7$ & $\begin{array}{c}1.043,0 \mathrm{~b} \\
(1,0)\end{array}$ \\
\hline Jul & $\begin{array}{c}6.625 .196,6 \\
(6,2)\end{array}$ & $\begin{array}{l}105,1 \\
(12,1)\end{array}$ & $63.037,1$ & $\begin{array}{c}1.012,7 \\
(6,2)\end{array}$ & 2018 & $\begin{array}{c}6.622 .453,4 \mathrm{ab} \\
(1,5)\end{array}$ & $\begin{array}{c}121,2 \mathrm{a} \\
(3,6)\end{array}$ & $54.640,7$ & $\begin{array}{c}1.021,3 \mathrm{~b} \\
(3,9)\end{array}$ \\
\hline Ago & $\begin{array}{c}6.679 .461,1 \\
(5,9)\end{array}$ & $\begin{array}{l}105,9 \\
(12,2)\end{array}$ & $63.073,3$ & $\begin{array}{c}1.016,9 \\
(6,0)\end{array}$ & Taxa & $-0,26$ & 5,50 & & 2,37 \\
\hline Set & $\begin{array}{c}6.683 .227,6 \\
(6,4)\end{array}$ & $\begin{array}{l}105,7 \\
(11,9)\end{array}$ & $63.228,3$ & $\begin{array}{c}1.022,1 \\
(6,2)\end{array}$ & & & & & \\
\hline Out & $\begin{array}{c}6.594 .230,7 \\
(5,7)\end{array}$ & $\begin{array}{l}106,4 \\
(10,1)\end{array}$ & $61.975,9$ & $\begin{array}{c}1.024,6 \\
(5,8)\end{array}$ & & & & & \\
\hline Nov & $\begin{array}{c}6.682 .598,7 \\
(6,2)\end{array}$ & $\begin{array}{l}107,4 \\
(10,8)\end{array}$ & $62.221,6$ & $\begin{array}{c}1.027,0 \\
(5,4)\end{array}$ & & & & & \\
\hline Dez & $\begin{array}{c}6.603 .208,9 \\
(8,0)\end{array}$ & $\begin{array}{l}108,1 \\
(9,9)\end{array}$ & $61.084,3$ & $\begin{array}{c}1.029,4 \\
(5,3)\end{array}$ & & & & & \\
\hline
\end{tabular}

em que: Méd = Média; $\mathrm{CV}_{\%}=$ Coeficiente de variação em porcentagem; $\mathrm{CoC}=$ Cadeia de custódia e $\mathrm{N}=$ Número

Obs.: As médias seguidas por uma mesma letra, em coluna, não diferem entre si a nível de 5\% de significância pelo teste de Tukey.

A Figura 1 mostra a distribuição mensal e anual, em média, para empreendimentos certificados FSC em manejo florestal (área e número) e cadeia de custódia florestal (número).

O baixo coeficiente de variação apresentado, tanto para manejo florestal como cadeia de custódia, indica que os empreendimentos mantêm estabilidade entre os anos de 2012 a 2018, tendo, em média, valores máximos, para manejo florestal (área e número) e cadeia de custódia (número), respectivamente, 14,5; 4,8; e 3,9 \%. Já para o coeficiente de variação mensal, também foram apresentados valores, em média, considerados baixos, respectivamente, em seus valores máximos, 14,$1 ; 13,0$; e 9,4\%.
Em relação a comparação mensal, as áreas manejadas certificadas e em cadeia de custódia não apresentaram diferenças significativas entre si. Independentemente do mês avaliado, as médias foram semelhantes, variando de 6.303.503,9 a 6.683.227,6 ha, em um número de 101,3 a 108,1 empreendimentos certificados em manejo florestal. (Tabela 1).

A relação área/número para empreendimentos com certificação em manejo florestal apresentou uma variação de 60.823,5 ha (abril) até 63.228,3 ha (setembro), ou seja, 2.404,80 ha maior. Para a relação área/número anual, oscilou de 54.640,7 ha (2018) a $76.529,2$ ha (2014), resultando em uma área menor, cerca de $21.888,5$ ha. 

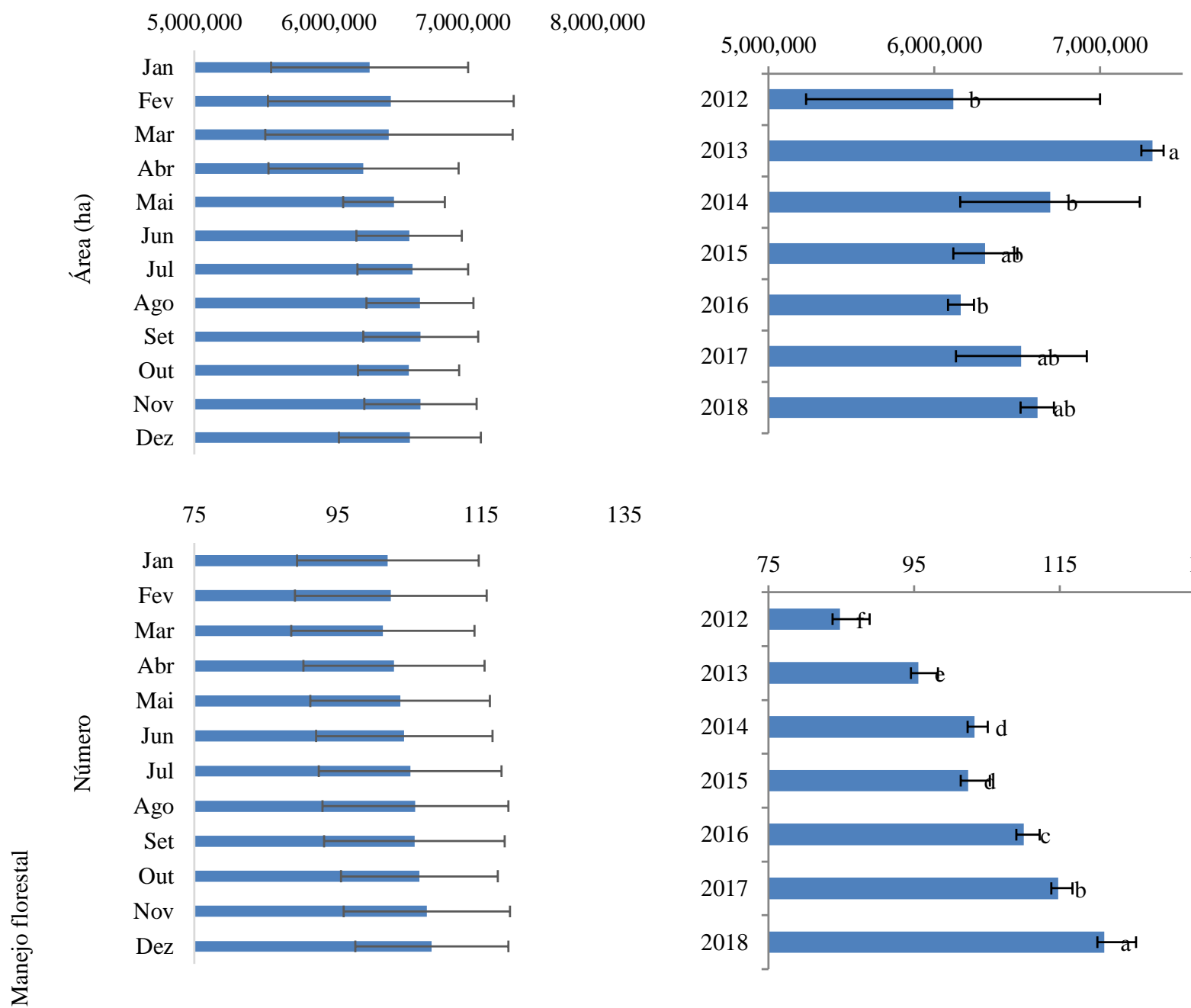

135
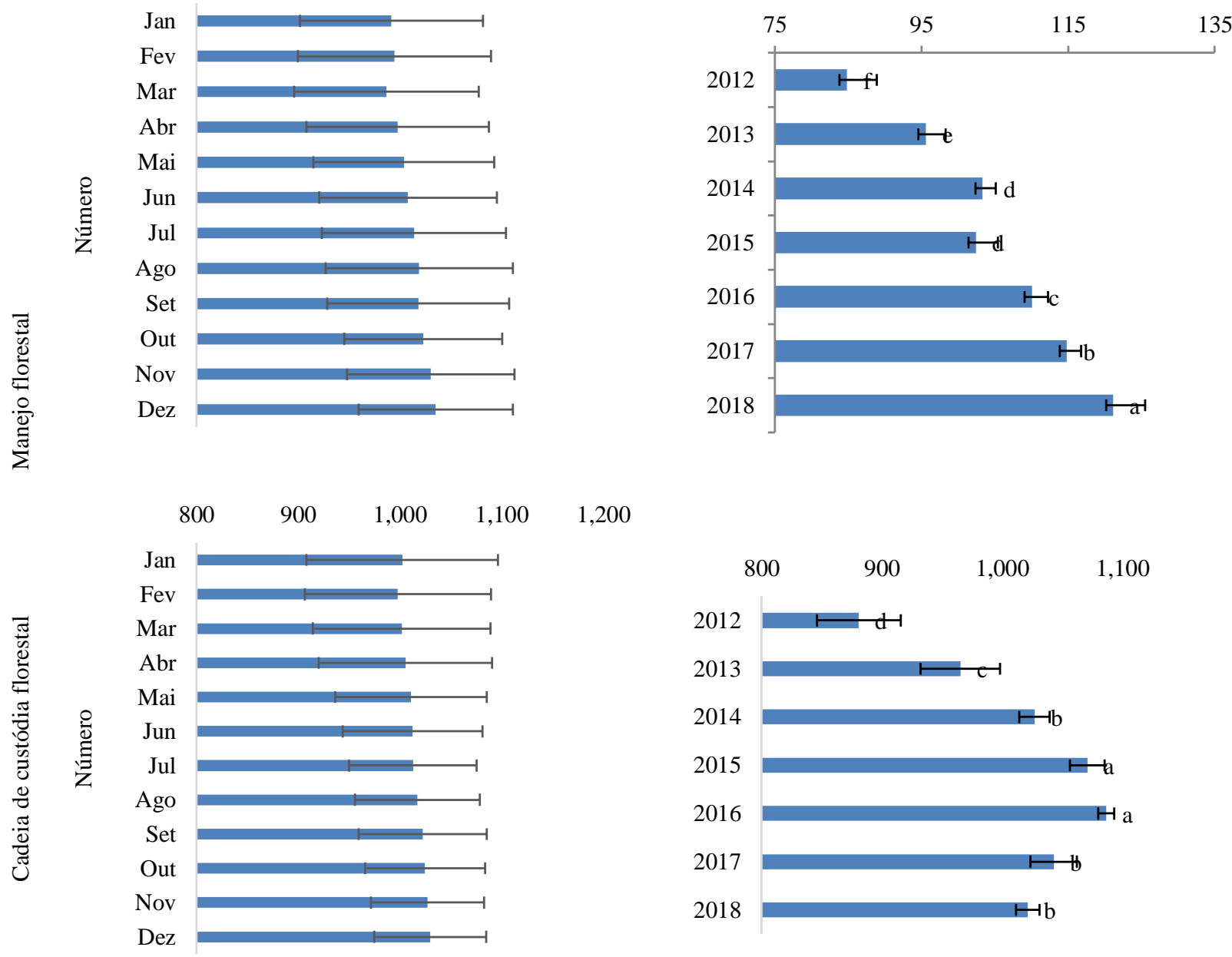

1,200

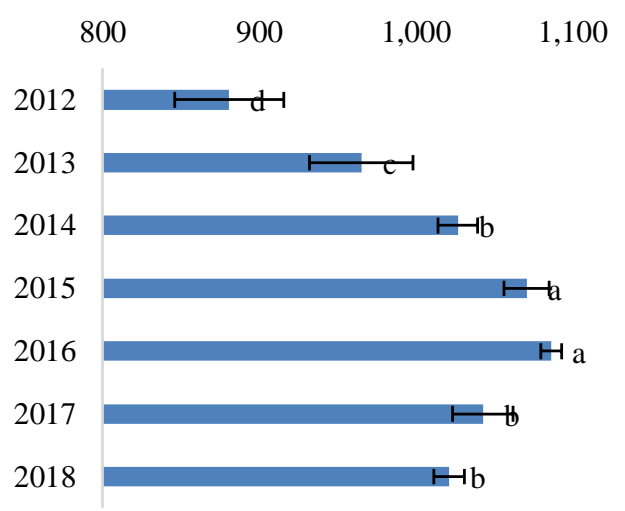

Figura 1- Distribuição, em média, para empreendimentos certificados FSC em manejo florestal e cadeia de custódia florestal. Obs.: As médias seguidas por uma mesma letra, em coluna, não diferem entre si a nível de 5\% de significância pelo teste de Tukey.

A área dos empreendimentos certificados em manejo florestal anual apresentou diferença significativa a nível de 5\% pelo teste de Tukey entre o período de 2012 a 2018. As maiores áreas certificadas em manejo florestal ocorreram nos anos de 2013, 2015, 2017 e 2018. Em relação ao número de empreendimentos certificados em manejo florestal foi apresentado crescimento de 2014 a 2018. A cadeia 
de custódia certificada foi maior nos anos de 2015 e 2016.

De janeiro a julho de 2019, o Brasil possuía 7.010.025,6 ha certificados, já ultrapassando o somatório de todo ano de 2018 quanto áreas certificadas em manejo florestal (MEIJUEIRO et al., 2020). O mesmo autor descreve os principais ramos em que a certificação em cadeia de custódia foi aplicada, sendo celulose e papel, mobiliário de interiores, produtos de madeira maciça, produtos de madeira para construção, painéis, e mobiliário de exterior e jardim.

As regiões sul e sudeste foram as maiores detentoras de certificados, enquanto a região nordeste, menor quantitativo. São Paulo foi o Estado que exibiu mais certificados na modalidade de cadeia de custódia (SANTOS, PELISSARI e SANQUETTA, 2017).

\section{CONCLUSÕES}

A certificação florestal é uma ferramenta eficaz para comprovação de manejos florestais viáveis, que se preocupam com o meio ambiente e a sociedade. Pode-se concluir que, no período de 2012 a 2018:

Entre os meses avaliados não houve diferença significativas para os empreendimentos certificados em manejo florestal e em cadeia de custódia.

Quando se comparou as variáveis anualmente, houve diferença significativa entre as mesmas. Para a área florestal manejada, em média, as maiores foram relacionais aos anos de 2013, 2015, 2017 e 2018. Para o número de certificados em manejo florestal, em média, se concentrou em 2018. E para o número de certificados em cadeia de custódia, no período de 2015 a 2016.

Houve crescimento no número de empreendimentos certificados FSC em manejo florestal e cadeia de custódia, no entanto, há uma redução no tamanho das áreas manejadas.

\section{REFERÊNCIAS}

ALMEIDA, A. N.; SANTOS, A. J.; SILVA, J. C. G. L.; BITTENCOURT, A. M. Análise do mercado dos principais produtos não-madeiráveis do estado do Paraná. Floresta, Curitiba, v.39, n.4, p.753-763, 2009.

AGUIAR, G. P.; ROCHA, J. D. S.; SANTOS, A. J.; SILVA, J. C. G. L.; HOEFLICH, V. A. Comportamento do mercado dos principais produtos florestais não madeireiros da região nordeste do Brasil. Enciclopédia Biosfera, Goiânia, v. 10, n. 18, p. 983-992, 2014.

BASSO, V. M.; JACOVINE, L. A. G.; ALVES, R. R.; VIEIRA, S. L. P. Influência da certificação florestal no cumprimento da legislação ambiental e trabalhista na região amazônica. Acta Amazonica. V. 41, n. 1, p. 69-76, 2011a.

SANTOS, T. W.; PELISSARI, A. L.; SANQUETTA, C. R. Quantificação e distribuição espacial dos certificados
BASSO, V. M.; JACOVINE, L. A. G.; ALVES, R. R.; VALVERDE, S. R.; SILVA, E. L.; BRIANEZI, D. Avaliação da influência da certificação florestal no cumprimento da legislação ambiental em plantações florestais. Revista Árvore. Viçosa, v. 35, n. 4, p. 835-844, $2011 b$.

BASSO, V. M.; JACOVINE, L. A.; ALVES, R. R.; VIERIA, S. L. P.; SILVA, F. L. Certificação florestal em grupo no Brasil. Floresta e Ambiente. Seropédica, v. 18, n. 2, p. 160-170, 2011c.

FOREST STEWARDSHIP COUNCIL. Disponível em: $<$ https://br.fsc.org/pt-br/fsc-brasil/fatos-e-nmeros> Acesso em: $\langle 14 / 07 / 2021>$.

GUJARATI, D. Econometria básica. 4 ed. Rio de Janeiro: Elsevier, 2006.

GUIMARAES, P. P.; BOTREL, R. T.; NOGUEIRA, N. W.; CASTRO, V. G.; AGUIAR, G. P.; CARMO, F. C. A. Produtos florestais não madeireiros do nordeste brasileiro: carnaúba. Nativa, Sinop, v. 6, n. 2, p. 213-218, 2018.

IBGE - Instituto Brasileiro de Geografia e Estatística. Disponível em: <http://www.sidra.ibge.gov.br/bda/pesquisas/pevs/default. asp> Acesso em: 07/06/2021.

MEIJUEIRO, D. V. M.; LOPES, C. S.; ALVES, R. R.; SILVEIRA, B. D.; GRACIOLI, C. R. Cerificação em manejo florestal e em cadeia de custódia no Brasil. Brazilian Journal of Development. v. 6, n. 8, p. 5732457340, 2020.

PAIVA, S. N.; SILVA, D. A.; ROCHADELLI, R.; HOSOKAWA, R. T.; OSHIRO, C. R. A certificação florestal pelo FSC: um estudo de caso. Floresta. V. 45, n. 2, p.213-222, 2015.

PEREIRA, L. C.; PELISSARI, A. L.; SANQUETTA, C. R.; EBLING, A. A. Estudo de caso da adequação de uma serraria às normas FSC de cadeia de custódia. Biofix Scientific Journal. v. 2, n. 1, p. 7-15, 2017.

PORTAL DA EDUCAÇÃO - Certificação Florestal. Disponível em: <https://www.portaleducacao.com.br/cursos/psicologia2/curso-livre/biologia/certificacao-florestal/714> Acesso em: $<14 / 07 / 2021>$

SPATHELF, P.; MATTOS, P. P.; BOTOSSO, P. C Certificação florestal no Brasil - uma ferramenta eficaz para conservação das florestas naturais. Floresta. v. 34, n. 3, p. 373-379, 2004.

TECPAR - Regulamento para certificação de cadeia de custódia para produtos de base florestal. Disponível em: $<$ http://tecpar.br/cert/quest/Regulamento_CadCustodia.pd f $>$. Acesso em: $\langle 14 / 07 / 2021\rangle$.

florestais FSC no Brasil. Enciclopédia Biosfera. v. 4, n. 8, p. 228-239, 2017. 